\title{
Energy Calibration of the NewSUBARU Storage Ring for Laser Compton-Scattering Gamma Rays and Applications
}

Hiroaki Utsunomiya, Tatsushi Shima, Keiji Takahisa, Dan Mihai Filipescu, Ovidiu Tesileanu, Ioana Gheorghe, Hilde-Therese Nyhus, Therese Renstrøm, Yiu-Wing Lui, Yasuhisa Kitagawa, Sho Amano, and Shuji Miyamoto

\begin{abstract}
Using $\gamma$-ray beams produced in the inverse Compton scattering between $\mathrm{CO}_{2}$ laser photons and relativistic electrons, we have calibrated electron beam energies in the nominal energy range 550-974 $\mathrm{MeV}$ at the synchrotron radiation facility NewSUBARU. The laser Compton-scattering (LCS) $\gamma$-ray beams were produced at energies from 561 to $1728 \mathrm{keV}$ and detected with a high-purity germanium detector. The electron beam energies were determined by reproducing the full energy peaks of the $\gamma$-ray beams by Monte Carlo simulations. The accuracy of the calibration is $(5.5-9.4) \times 10^{-5}$. The reproducibility of the electron beam energy is excellent in an independent injection and deceleration. The present energy calibration of the electron beams offers a standard for the energy calibration of high-energy LCS $\gamma$-ray beams produced with a $\mathrm{Nd}: \mathrm{YVO}_{4}$ laser. As applications of the energy calibration, we investigated the energy linearity of a $3.5^{\prime \prime} \times 4.0^{\prime \prime} \mathrm{LaBr}_{3}(\mathrm{Ce})$ detector in the response to $\gamma$-rays at energies up to $10 \mathrm{MeV}$ and the energy profile of the high-energy LCS $\gamma$-ray beams.
\end{abstract}

Index Terms-Electron accelerators, gamma-ray detection, storage ring.

Manuscript received January 10, 2014; revised February 14, 2014 and March 03, 2014; accepted March 13, 2014. Date of publication May 20, 2014; date of current version June 12, 2014. This work was supported by the Japan Private School Promotion Foundation and partly by the JSPS-FNRS bilateral program. D. M. Filipescu, O. Tesileanu, and I. Gheorghe acknowledge financial support from the Extreme Light Infrastructure Nuclear Physics (ELI-NP) Phase I, a project co-financed by the European Union through the European Regional Development Fund. H.-T. Nyhus and T. Renstrøm acknowledge financial support from the Norwegian Research Council (NFR), project number 210007

H. Utsunomiya is with the Department of Physics, Konan University, Higashinada, Kobe 658-8501, Japan, and the Center for Nuclear Study, University of Tokyo, Wako, Saitama 351-0198, Japan (e-mail: hiro@ konan-u.ac.jp).

T. Shima and K. Takahisa are with the Research Center for Nuclear Physics, Osaka University, Suita, Osaka 567-0047, Japan.

D. M. Filipescu is with the Extreme Light Infrastructure Nuclear Physics and the National Institute for Physics and Nuclear Engineering Horia Hulubei, Bucharest-Magurele 077125, Romania

O. Tesileanu is with the Extreme Light Infrastructure Nuclear Physics, Bucharest-Magurele 077125, Romania.

I. Gheorghe is with the National Institute for Physics and Nuclear Engineering Horia Hulubei, Bucharest-Magurele 077125, Romania.

H. T. Nyhus and T. Renstrøm are with the Department of Physics, University of Oslo, N-0316 Oslo, Norway.

Y.-W. Lui is with the Cyclotron Institute, Texas A\&M University, College Station, Texas 77843 USA.

Y. Kitagawa, S. Amano, and S. Miyamoto are with the Laboratory of Advanced Science and Technology for Industry, University of Hyogo, Kamigori, Ako-gun, Hyogo 678-1205, Japan.

Color versions of one or more of the figures in this paper are available online at http://ieeexplore.ieee.org.

Digital Object Identifier 10.1109/TNS.2014.2312323

\section{INTRODUCTION}

Q UASIMONOCHROMATIC $\gamma$-ray beams are produced at the synchrotron radiation facility NewSUBARU in the inverse Compton scattering of laser photons from relativistic electrons circulating in a storage ring [1], [2]. A beam of $974 \mathrm{MeV}$ electrons is injected from a linear accelerator into the NewSUBARU storage ring. The energy represents a relative value with $\sim 1 \%$ uncertainty based on the magnetic field strength and the beam optics of the storage ring [3] and is hereafter referred to as the nominal energy in this paper. The injected beam can be either decelerated down to $0.5 \mathrm{GeV}$ or accelerated up to $1.5 \mathrm{GeV}$.

In the laser inverse Compton scattering, the energy of scattered photons is given by

$$
E_{\gamma}=\frac{4 \eta^{2} \varepsilon_{L}}{1+(\eta \theta)^{2}+4 \eta \varepsilon_{L} /\left(m c^{2}\right)}
$$

where $\varepsilon_{L}$ is the energy of a laser photon, $m c^{2}$ is the rest mass energy of an electron, $\theta$ is the scattering angle of a laser photon with respect to the electron incident direction, and $\eta$ is the Lorentz factor for electron, $\eta=E_{e} / m c^{2}$, defined by the total electron energy $E_{e}$ and the rest mass energy.

The energy amplification factor in nearly head-on collisions $(\theta \approx 0), E_{\gamma} / \varepsilon_{L} \approx 4 \eta^{2}$, is very large on the order of $10^{6}-10^{7}$ for several hundred megaelectronvolts $(\mathrm{MeV})$ to a few gigaelectronvolts $(\mathrm{GeV})$ electrons $\left(1.6 \times 10^{7}\right.$ for $\left.E_{e}=1 \mathrm{GeV}\right)$ so that an $\mathrm{eV}$ laser beam can be converted to an $\mathrm{MeV} \gamma$-ray beam in the laser inverse Compton scattering. Note that the numerator in (1) is a dominating factor to determine the LCS $\gamma$-ray energy. At NewSUBARU, one can produce low-energy $\gamma$-ray beams at a few megaelectronvolts using a $\mathrm{CO}_{2}$ laser (wavelength $\lambda=$ $10.59 \mu \mathrm{m}$ ) and high-energy $\gamma$-ray beams at a few tens of megaelectronvolts using a Nd: $\mathrm{YVO}_{4}$ laser $(\lambda=1064 \mathrm{~nm})$ in collisions with $0.5-1.0 \mathrm{GeV}$ electrons.

It is not straightforward to calibrate the energy of the LCS $\gamma$-ray beam in the region of a few tens of megaelectronvolts for a lack of proper $\gamma$-ray sources. A high-resolution germanium detector may be used for calibration. However, the standard $\gamma$-ray sources can cover only low energies so that a linear extrapolation must be employed to extend the energy range to high energies. It may not be surprising that such extrapolation causes uncertainties of $\sim 50 \mathrm{keV}$ around $10 \mathrm{MeV}$. Furthermore, a large-volume Ge detector is needed to detect a high-energy 
edge corresponding to the full-energy (FE) peak for $10 \mathrm{MeV}$ $\gamma$-rays if it may not identify the FE peak as an isolated peak.

One can see by setting $\theta=0$ in (1) that the maximum energy of the LCS $\gamma$-ray beam can be determined if the electron beam energy is known. It is, however, risky to rely on the nominal energy because the $1 \%$ uncertainty results in $2 \%$ uncertainty in the maximum $\gamma$-ray energy. Obviously, one derives from (1), the following:

$$
\frac{\Delta E_{\gamma}}{E_{\gamma}} \approx 2 \frac{\Delta E_{e}}{E_{e}}
$$

We searched the neutron threshold for ${ }^{197} \mathrm{Au}$ located at $8.07 \mathrm{MeV}$ [4] by detecting neutrons from ${ }^{197} \mathrm{Au}(\gamma, n)$ reactions with a 2 -mm-thick ${ }^{197} \mathrm{Au}$ target. LCS $\gamma$-ray beams were produced with a $\mathrm{Nd}: \mathrm{YVO}_{4}$ laser and electron beams at the nominal electron energies from 677 to $661 \mathrm{MeV}$ in steps of $2 \mathrm{MeV}$. By changing the electron-beam energy by $2 \mathrm{MeV}$, we changed the $\gamma$-ray energy by $\sim 50 \mathrm{keV}$. Note that electrons at $676.43 \mathrm{MeV}$ produce an LCS $\gamma$-ray beam with the maximum energy at the neutron threshold for ${ }^{197} \mathrm{Au}$. Neutrons were detected with a $4 \pi$ neutron detector consisting of $20{ }^{3} \mathrm{He}$ proportional counters embedded in a polyethylene moderator. Statistically significant events were observed above the nominal energy $667 \mathrm{MeV}$, indicating that the neutron threshold lies between two LCS $\gamma$-ray energies produced by electrons at the nominal energies of 667 and $665 \mathrm{MeV}$. This observation led to the fact that there is a $1.4 \%-1.7 \%$ discrepancy between real and nominal energies in this energy region; the real energy is higher than the nominal energy.

The threshold search for ${ }^{197} \mathrm{Au}$ showed that absolute calibration of electron beam energies is of critical importance to photoneutron cross-section measurements near neutron threshold with LCS $\gamma$-ray beams. We performed absolute calibration of electron beam energies of the storage ring NewSUBARU in the nominal energy range of $0.55-1.0 \mathrm{GeV}$. In this paper, we report results on the calibration of the NewSUBARU and applications of calibrated electron beams.

\section{EXPERIMENT}

The technique of laser Compton backscattering has been developed to accurately determine electron beam energies as an alternative to the technique of the resonant spin depolarization, which is limited to high energy electrons because of the spin depolarization time [6]. This technique was widely used to calibrate electron beam energies by using low-energy LCS $\gamma$-ray beams produced with a $\mathrm{CO}_{2}$ laser [5], [7], [8], [9] and a free electron laser [10] at different facilities worldwide. Unlike the past calibrations, however, we performed the energy calibration systematically in the following five steps:

1) production of low-energy LCS $\gamma$-ray beams in collisions of $\mathrm{CO}_{2}$ laser photons with electrons at ten nominal energies from 974 to $550 \mathrm{MeV}$;

2) measurements of LCS $\gamma$-ray beams with a high-purity germanium (HPGe) detector;

3) energy calibration of the HPGe detector with the standard $\gamma$-ray sources;

4) determination of electron beam energies by Monte Carlo simulations;
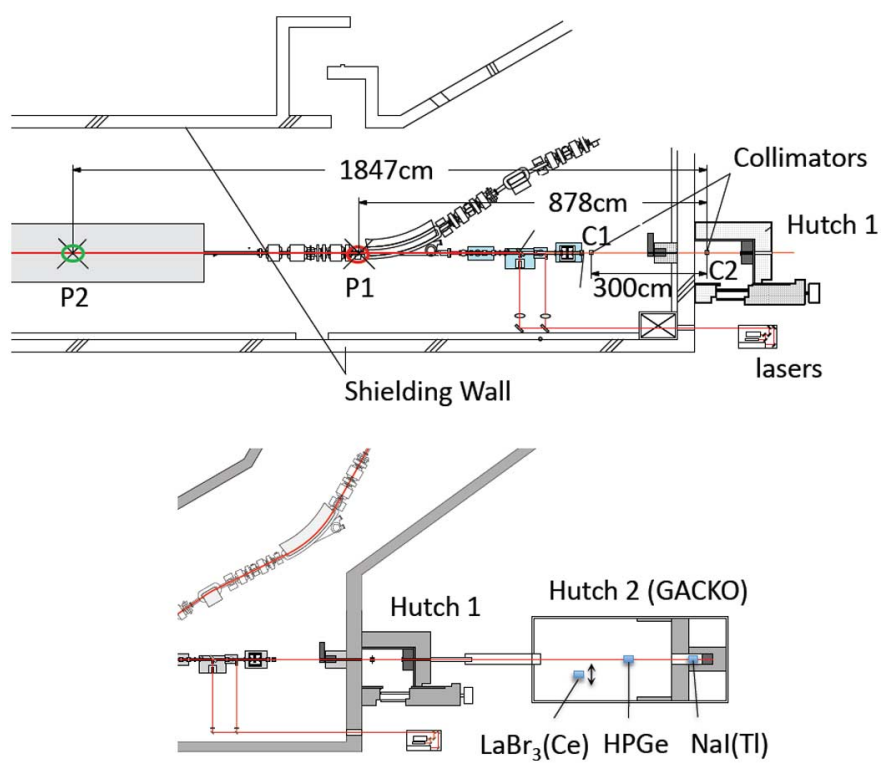

Fig. 1. Experimental setup.

5) study of the accuracy and the reproducibility of the electron beam energies.

Fig. 1 depicts the experimental setup for the present measurement in the $\gamma$-ray beam line BL01 of the NewSUBARU synchrotron radiation facility. An electron beam was injected from the linear accelerator into the NewSUBARU storage ring at the nominal energy $974 \mathrm{MeV}$. A grating-fixed $\mathrm{CO}_{2}$ laser (Infrared Instruments, San Marcos, CA, USA, IR-10-WS-GF-VP) oscillated at a single line of the strongest master transition $\mathrm{P}(20)$. The central wavelength of the $\mathrm{P}(20)$ transition is known $(\lambda=$ $10.5915 \mu \mathrm{m} \pm 3 \AA$ ) [11] with the bandwidth $1.3 \AA$ in the fullwidth at half-maximum (FWHM) [12]. The bandwidth, i.e., the gain linewidth $340 \mathrm{MHz}$ in FWHM, was provided by Infrared Instruments. Including the bandwidth, the accuracy of the wavelength of $\mathrm{CO}_{2}$ laser is $4.1 \times 10^{-5}$.

The $\mathrm{CO}_{2}$ laser photons produced outside the storage ring vault were led through four mirrors and one lens into the vacuum tube of the ring to a collision point $\mathrm{P} 1$ in the straight section of the storage ring. The alignment and stability of the laser optical elements were carefully checked by sending a $\mathrm{He}-\mathrm{Ne}$ laser beam into the straight section of the ring from the upstream on the other side of the beam line and back to the center of the exit of the $\mathrm{CO}_{2}$ laser through the optical elements. The collision point $\mathrm{P} 1$ for the $\mathrm{CO}_{2}$ laser is located at the distance of $878 \mathrm{~cm}$ from a collimator set in the experimental Hutch 1 . The collision between laser photons and electrons takes place with the maximum collision efficiency at $\mathrm{P} 1$, where the electron beam forms a narrow waist in the beam optics [1]. In contrast, the collision point $\mathrm{P} 2$ for the $\mathrm{Nd}: \mathrm{YVO}_{4}$ laser is located at $1847 \mathrm{~cm}$ from the collimator.

The fundamental parameters for the production of low-energy LCS $\gamma$-ray beams such as the central wavelength $(\lambda)$ and the bandwidth $(\delta \lambda)$ of the $\mathrm{CO}_{2}$ laser, the size $\left(\sigma_{e}\right)$, the divergence $\left(\theta_{e}\right)$, and the energy spread $\left(\delta E_{e}\right)$ of the $974 \mathrm{MeV}$ electron beam, and the angle acceptance of the collimator $\left(\theta_{c}\right)$ are listed in Table I [1], [13]. The electron beam size at the collision 
TABLE 1

Fundamental Parameters for the Production of a Low-Energy LCS $\gamma$-RAY BEAM WITH A $\mathrm{CO}_{2}$ LASER AT THE NOMINAL ELECTRON BEAM ENERGY 974 MeV at the NewSUBARU Storage Ring: The Central Laser WAVELENGTH $\lambda$, THE LASER BANDWidTh $\delta \lambda$, THE Electron BeAm Size $\sigma_{e}$, THE Electron BeAm Divergence $\theta_{\epsilon}$, THE Electron BeAm EnERGy SPREAD $\delta E_{e}$, AND THE ANGLE ACCEPTANCE OF THE $2 \mathrm{~mm}$ COLlimator $\theta_{c}$

\begin{tabular}{cccccc}
\hline \hline $\begin{array}{c}\text { central } \lambda \\
(\mu \mathrm{m})\end{array}$ & $\begin{array}{c}\text { band width } \delta \lambda \\
\text { FWHM }\end{array}$ & $\begin{array}{c}\sigma_{e} \\
(\mathrm{~mm})\end{array}$ & $\begin{array}{c}\theta_{e} \\
(\mathrm{mrad})\end{array}$ & $\begin{array}{c}\delta E_{e} \\
(\%)\end{array}$ & $\begin{array}{c}\theta_{c} \\
(\mathrm{mrad})\end{array}$ \\
\hline $10.5915 \pm 3 \AA$ & $1.3 \AA$ & 0.3 & 0.13 & 0.042 & 0.11 \\
\hline
\end{tabular}

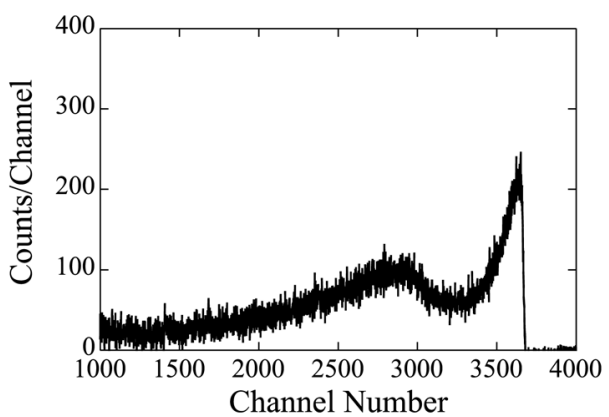

Fig. 2. Response function of the HPGe detector to LCS $\gamma$-rays produced in collisions of $\mathrm{CO}_{2}$ laser photons with electrons at the nominal energy of $850 \mathrm{MeV}$.

point $\mathrm{P} 1$, which is circular, is calculated from the Twiss parameters in the straight section of the ring [1].

The beam line BL01 has two collimators; one $(\mathrm{C} 1)$ in the storage ring vault and the other (C2) in Hutch 1. Both collimators are made of 10 -cm-thick $\mathrm{Pb}$. In the present experiment, we used the $\mathrm{C} 1$ collimator with an aperture of $6-\mathrm{mm}$ diameter. The $\mathrm{C} 2$ collimator with an aperture of 2-mm diameter defined scattering angle for laser Compton backscattering. An $\mathrm{NaI}(\mathrm{Tl})$ detector of 6-in diameter and 5-in thickness was mounted at the end of the beam line in the experimental Hutch 2, GACKO (Gamma collaboration hutch of Konan University), to monitor the $\gamma$-ray flux. The $\mathrm{C} 2$ collimator was mounted on an $x-y-\theta$ stage driven by stepping motors. Finetunings of the laser optics and the collimator alignment along the horizontal $(x)$, vertical $(y)$, and rotational $(\theta)$ axes were carried out to maximize the $\gamma$-ray flux. A coaxial HPGe detector (64 $\mathrm{mm}$ in diameter $\times 60 \mathrm{~mm}$ in length) was mounted in GACKO and aligned with synchrotron radiation to measure the low-energy LCS $\gamma$-rays. The HPGe detector was calibrated with the standard $\gamma$-ray sources, ${ }^{60} \mathrm{Co}$ including the sum peak, ${ }^{133} \mathrm{Ba},{ }^{137} \mathrm{Cs}$, and ${ }^{152} \mathrm{Eu}$ and a natural radioactivity ${ }^{40} \mathrm{~K}$.

After the injection of $974 \mathrm{MeV}$ electrons into the NewSUBARU storage ring, the electron beam was decelerated to the nominal energy $950 \mathrm{MeV}$, and subsequently down to $550 \mathrm{MeV}$ in steps of $50 \mathrm{MeV}$ followed by a production of the LCS $\gamma$-ray beam and a measurements with the HPGe detector at every energy.

\section{RESULTS}

\section{A. Response Functions of the HPGe Detector to LCS $\gamma$-ray Beams}

Fig. 2 shows a response function of the HPGe detector to LCS $\gamma$-rays produced in collisions of $\mathrm{CO}_{2}$ laser photons with electrons at the nominal energy of $850 \mathrm{MeV}$. One can identify the FE peak as well as the Compton scattered component. The FE peak has an energy spread arising from angle acceptance of the 2-mm collimator and the electron beam emittance. Furthermore, the high-energy edge of the FE peak has a slope that reflects the energy resolutions of the electron beam and the HPGe detector. Because of the energy spread and the slope of the FE peak, the electron beam energy cannot be determined analytically using (1).

\section{B. Energy Calibration}

The production of LCS $\gamma$-rays and the response function of the HPGe detector to the $\gamma$-rays were simulated with the Monte Carlo code of [8]. The EGS4/PRESTA code simulates laser-Compton backscattering and interactions of $\gamma$-rays with detector materials. The production of $\gamma$-rays is based on the kinematics and the cross section for laser Compton backscattering. The cross section in the rest frame of electron is given by the Klein-Nishina formula. The code EGS4 [14] is utilized for the latter simulation.

The effect of the electron beam emittance on the laser Compton backscattering consists of those of the beam size and the beam divergence. The divergence of nonparallel electron beams causes a kinematical effect that increases an angular acceptance of the collimator for Compton backscattering compared with that for parallel beams and has no influence on the maximum energy of the LCS $\gamma$-ray beam, which geometrically corresponds to $\theta=0$. The electron beam size defines the size of "a light source" from which LCS $\gamma$-rays are emitted within a finite solid angle subtended by the collimator. Therefore, the angular acceptance of the collimator increases with increasing the electron beam size compared with that for the point source. As a result, the divergence and size of the electron beam have the same kinematical effect, which affects only the energy spread of the LCS $\gamma$-ray beam in the Monte Carlo simulation. Thus, the electron beam divergence can be effectively incorporated into the electron beam size, although they are different physics quantities, which forms an effective source size [15].

The divergence of the laser beam as well as of the electron beam does not change the maximum energy of the LCS $\gamma$-ray beam because it is distributed around zero approximately in a Gaussian function. The maximum allowed misalignment of the laser optics is estimated to be $3 \mathrm{mrad}$ for the lens with the Rayleigh length $40 \mathrm{~cm}$ used for the $\mathrm{CO}_{2}$ laser with the beam radius $1.2 \mathrm{~mm}$. The $3 \mathrm{mrad}$ misalignment between the incoming $\mathrm{CO}_{2}$ laser and $974 \mathrm{MeV}$ electron beam decreases the maximum energy of the LCS $\gamma$-ray beam by $3.3 \times 10^{-6}$. Therefore, laser misalignment, particularly after tuning the laser optics in the head-on collision geometry by optimizing the intensity of the LCS $\gamma$-ray beam, can be safely neglected in the present measurement.

The energy resolution of the HPGe detector can be effectively incorporated into the energy spread of the electron beam, which forms the effective energy spread. The two fitting parameters, the electron beam energy and the effective energy spread, have a good control on the position and the slop of the high energy edge of the HPGe response function, respectively, as shown in [6], whereas the effective source size has a good control on the energy spread of the LCS $\gamma$-ray beam. 

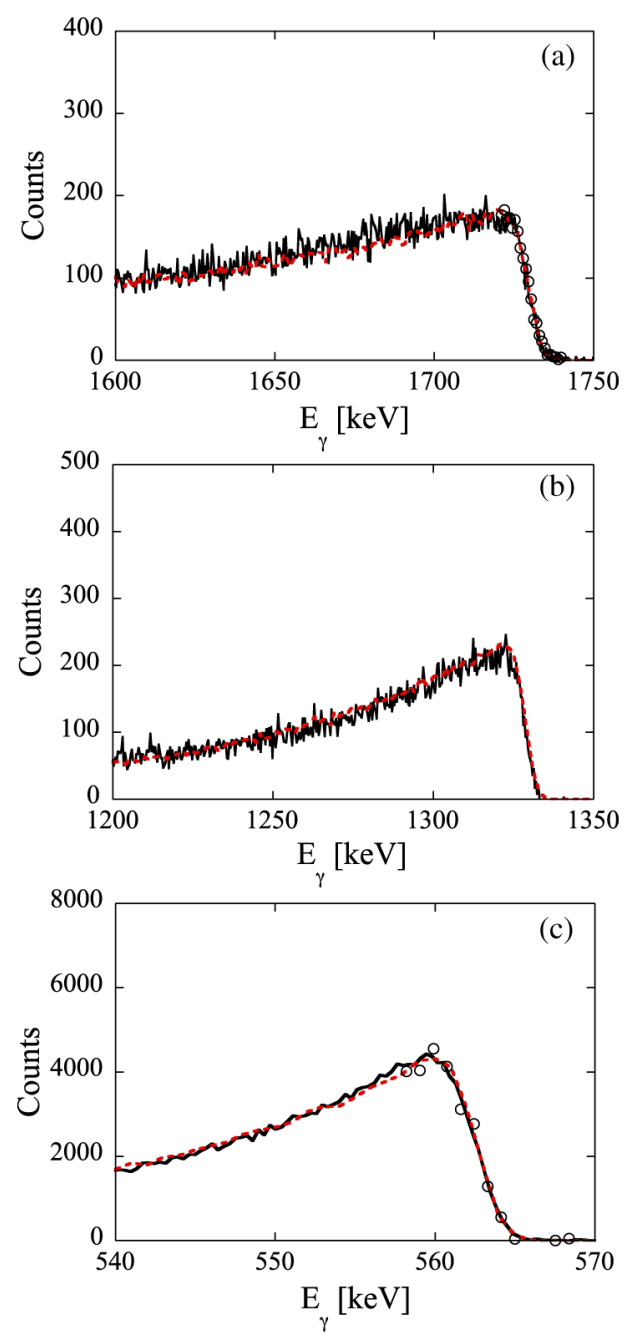

Fig. 3. (a) Monte Carlo simulation (dotted line) with a calibrated energy of the electron beam $982.2 \mathrm{MeV}$ in comparison with the experimental data (solid line) taken at a nominal energy $974 \mathrm{MeV}$. (b) The same as (a), but for a simulation with a calibrated energy $860.7 \mathrm{MeV}$ in comparison with the data at a nominal energy $850 \mathrm{MeV}$. (c) The same as (a), but for a simulation with a calibrated energy $559.6 \mathrm{MeV}$ in comparison with the data at a nominal energy $550 \mathrm{MeV}$.

Monte Carlo simulations were carried out to reproduce the FE peak of the response function with three fitting parameters: the electron beam energy, the effective energy spread, and the effective source size $(\zeta)$ at the collision point. The parameters of the distance of the collimator from the collision point, the collimator size, the wavelength of the $\mathrm{CO}_{2}$ laser, and the size of the HPGe crystal are fixed.

Examples of the best fits to the spectra are shown in Fig. 3. The FE peaks were well reproduced by the Monte Carlo simulations with the source size $\zeta=0.9-1.1 \mathrm{~mm}$. The overall energy resolution was taken to be $\Delta E_{e} / E_{e} \approx 0.1 \%$ in the standard deviation of Gaussian function, which is a quadratic sum of the detector resolution $0.077 \%$ ( $2.43 \mathrm{keV}$ in FWHM for $1332 \mathrm{keV}$ $\gamma$-rays) and the intrinsic energy spread of the electron beam $0.063 \%$. The estimated energy spread is consistent with the fundamental parameter listed in Table I.

The difference of the calibrated energy $E_{e}^{c}$, thus determined by the Monte Carlo simulation from the nominal energy $E_{e}^{n}$,

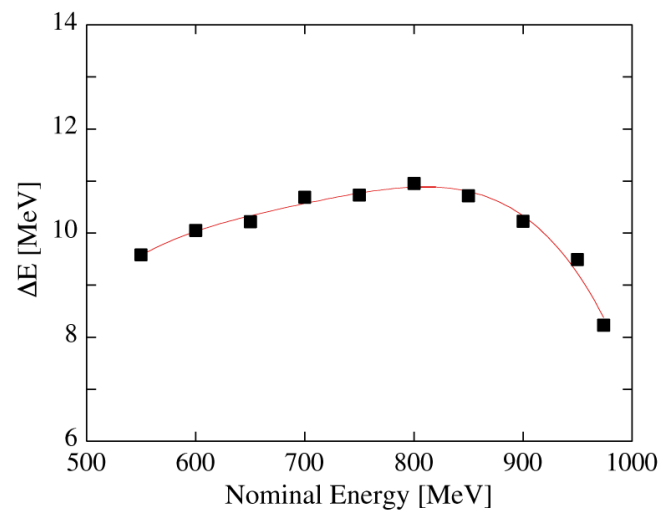

Fig. 4. Difference $\Delta E$ of the calibrated energy $E_{e}^{c}$ from the nominal energy $E_{e}^{n}$ of the electron beams, $\Delta E=E_{e}^{c}-E_{e}^{n}$, at the NewSUBARU storage ring.

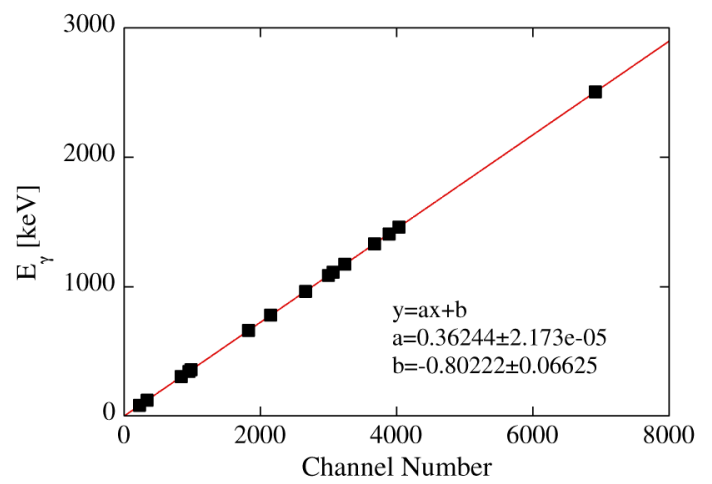

Fig. 5. Energy calibration of the HPGe detector with the standard $\gamma$-ray sources and a natural radioactivity ${ }^{40} \mathrm{~K}$ for a dynamic range up to the $2.5 \mathrm{MeV}$ sum peak of ${ }^{60} \mathrm{Co}$.

$\Delta E=E_{e}^{c}-E_{e}^{n}$ is shown in Fig. 4 as a function of $E_{e}^{n}$. The fourth-order polynomial fit to the data gives

$$
\begin{aligned}
\Delta E= & -4.6949 \times 10^{-10}\left(E_{e}^{n}\right)^{4}+1.3017 \times 10^{-6}\left(E_{e}^{n}\right)^{3} \\
& -1.3596 \times 10^{-3}\left(E_{e}^{n}\right)^{2}+0.63854\left(E_{e}^{n}\right)-103.94 .
\end{aligned}
$$

Here, $E_{e}^{c}$ and $E_{e}^{n}$ are given in megaelectronvolts. The discrepancy of the energy calculated by using (3) from the calibrated energy is $6-215 \mathrm{keV}\left(1 \times 10^{-3}-2 \times 10^{-2 \%}\right)$ at the ten data points. The difference between the nominal and calibrated energies is $10.44 \mathrm{MeV}(1.56 \%)$ at the nominal energy $667 \mathrm{MeV}$, which is consistent with the result of the neutron-threshold search for ${ }^{197} \mathrm{Au}$.

\section{Accuracy of the Electron Beam Energy}

Besides the wavelength of the $\mathrm{CO}_{2}$ laser, the accuracy of the electron beam energy is determined by two factors involved in the response function measurement, i.e., the energy calibration and the counting statistics of the response function [10]. The systematic uncertainty arises from the former factor and the wavelength through (1), while the statistical uncertainty arises from the latter factor in the least-squares fit to the response functions. The energy calibration of the HPGe detector is shown in Fig. 5. Note that the energy range of the LCS $\gamma$-ray beams produced below $1.8 \mathrm{MeV}$ is fully covered by the present calibration. The slope and intercept of the linear fit to the calibration data are given in the figure. The systematic uncertainty for the 


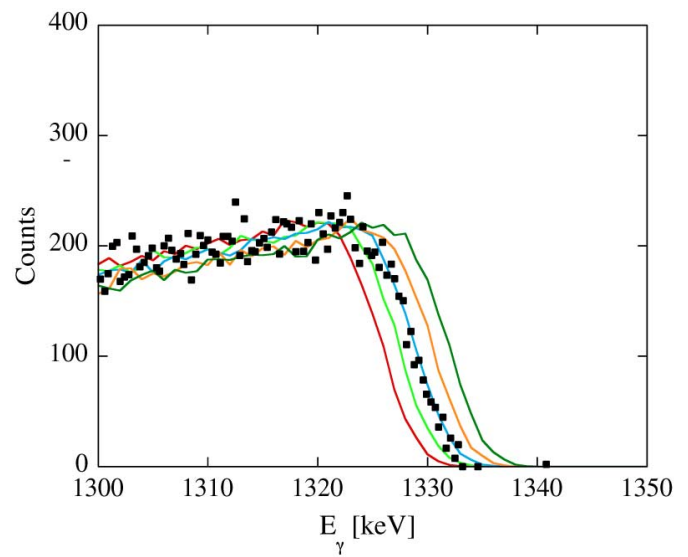

Fig. 6. Dependence of the Monte Carlo simulation on the electron beam energy is shown for the response function of the HPGe detector to the LCS $\gamma$-ray beam produced at the nominal energy $850 \mathrm{MeV}$. The best-fit result with $E_{\epsilon}^{c}=$ $860.72 \mathrm{MeV}$ is shown in comparison with those obtained with $E_{e}^{c}=860.72 \pm$ $0.50 \mathrm{MeV}$ and $E_{e}^{c}=860.72 \pm 1.00 \mathrm{MeV}$.

energy calibration is energy dependent; it is $7.9 \times 10^{-5}$ at the nominal energy $550 \mathrm{MeV}$ and $5.4 \times 10^{-5}$ at $974 \mathrm{MeV}$, including the accuracy of the wavelength $\left(4.1 \times 10^{-5}\right)$. We obtained the statistical uncertainty of $(1-5) \times 10^{-5}$ in the least-squares fit to the response functions. An example of the dependence of the Monte Carlo simulation on the electron beam energy is shown in Fig. 6. The overall uncertainty in the present calibration over $550-974 \mathrm{MeV}$ is $(5.5-9.4) \times 10^{-5}$, which is to be compared with the one $\left(3 \times 10^{-5}\right)$ reported in [10].

\section{Reproducibility of the Electron Beam Energy}

To test the reproducibility of the electron beam energy, we aborted the $550 \mathrm{MeV}$ electron beam left in the calibration runs, initialized all the dipole magnets of the storage ring, newly injected an electron beam at the nominal energy $974 \mathrm{MeV}$, and repeated the procedure of deceleration down to $550 \mathrm{MeV}$. We produced LCS $\gamma$-ray beams with the $\mathrm{CO}_{2}$ laser at two nominal energies, 974 and $550 \mathrm{MeV}$. The response functions of the HPGe detector measured in the second injection are shown by the open circles in Fig. 3(a) and (c). The high-energy edge of the LCS $\gamma$-ray beam was excellently reproduced at these two energies.

\section{APPLICATIONS}

\section{A. Linearity of a $\operatorname{LaBr}_{3}(\mathrm{Ce})$ Detector}

An interesting application of the present energy calibration of the electron beam may lie in energy calibration of photon detectors. To demonstrate such an application, we produced high-energy LCS $\gamma$-ray beams in an energy range of $10-8 \mathrm{MeV}$ at the nominal energies 753.0 (calibrated energy 764.0), 714.0 (724.8) and $677.0(687.7) \mathrm{MeV}$ with a $\mathrm{Nd}: \mathrm{YVO}_{4}$ laser (Spectra-Physics, Inazuma, $\lambda=1064 \mathrm{~nm}$ ) and measured the $\gamma$-ray beams with a $\mathrm{LaBr}_{3}(\mathrm{Ce})$ detector $\left(3.5^{\prime \prime}\right.$ in diameter $\times 4.0^{\prime \prime}$ in length, BrilLanCe380 89S102/3.5, Saint-Gobain) at the count-rate less than 10000 counts per second with a bias voltage of $+400 \mathrm{~V}$ and a shaping time $0.5 \mu \mathrm{s}$ of an NIM amplifier module. Fig. 7 shows response functions of the $\mathrm{LaBr}_{3}(\mathrm{Ce})$ detector to these LCS $\gamma$-rays.
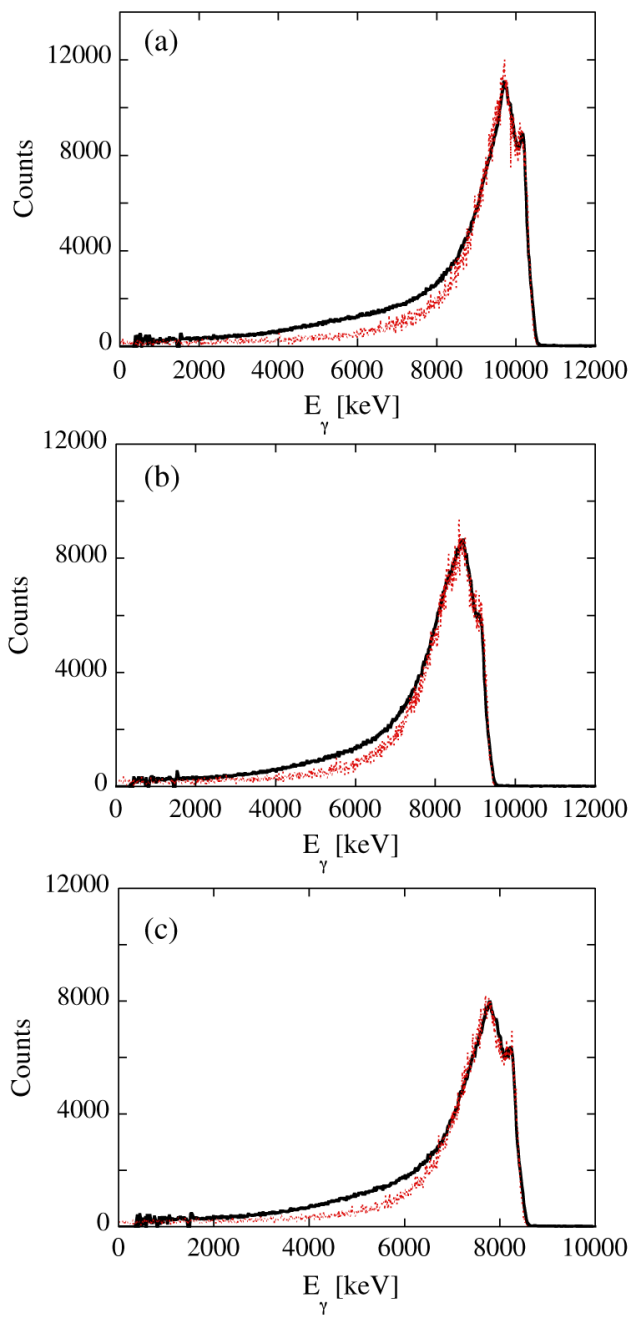

Fig. 7. (a) Monte Carlo simulations (dotted line) with $764.0 \mathrm{MeV}$ (nominal energy $753 \mathrm{MeV}$ ) and $\zeta=1.7 \mathrm{~mm}$ for the LCS $\gamma$-ray beam produced with a Nd: $\mathrm{YVO}_{4}$ laser in comparison with the experimental data (solid line). The experimental data are energy-calibrated following the simulation. (b) The same as (a), but for a simulation with $724.8 \mathrm{MeV}$ (nominal energy $714 \mathrm{MeV}$ ) and $\zeta=2.5 \mathrm{~mm}$. (c) The same as (a), but for a simulation with $687.7 \mathrm{MeV}$ (nominal energy $677 \mathrm{MeV}$ ) and $\zeta=2.5 \mathrm{~mm}$.

We performed Monte Carlo simulations of the response function of the $\mathrm{LaBr}_{3}(\mathrm{Ce})$ detector. The angle acceptance of the collimator with an aperture of 2-mm diameter, which is located at $1847 \mathrm{~cm}$ from the collision point $\mathrm{P} 2$, is $0.054 \mathrm{mrad}$. It is pointed out in [10] that when the $\gamma$ beam energy spread due to the collimation is smaller than or comparable to that due to the electron beam energy spread, the collimation effect will start to alter the high energy edge of the spectrum, resulting in a shift of the spectrum toward the higher energy. This is not the case in the present measurement because the $\gamma$ beam energy spread due to the collimation is $\eta^{2} \theta_{c}^{2}=(5.31-6.55) \times 10^{-3}$, whereas that due to the electron beam energy spread is $1.26 \times 10^{-3}$. The energy resolution of the $\mathrm{LaBr}_{3}(\mathrm{Ce})$ detector was incorporated into the simulation as effective energy resolution of the electron beam as was done for the HPGe detector. Best fits were obtained with the resolution $0.45 \%$, which can be translated into the detector resolution $2.1 \%$ in FWHM for $8-10 \mathrm{MeV} \gamma$-rays. The energy resolution of the present $\mathrm{LaBr}_{3}(\mathrm{Ce})$ detector was $4.0 \%$ in FWHM for $662 \mathrm{keV} \gamma$-rays and 2.6\% in FWHM for $1332 \mathrm{keV}$ 


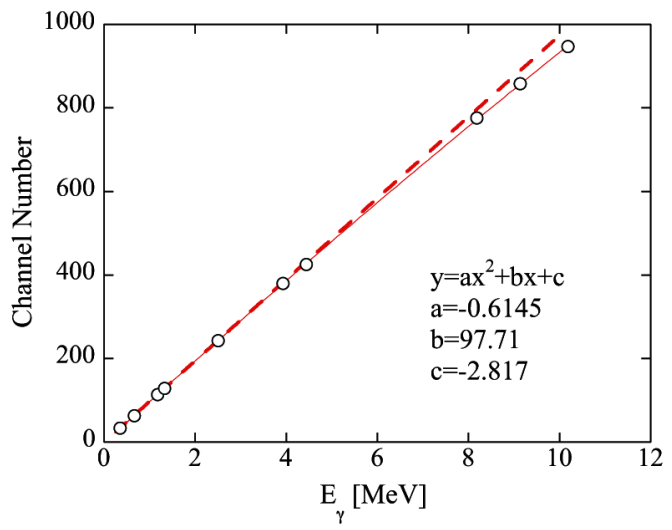

Fig. 8. Linearity in the response of a $\operatorname{LaBr}_{3}(\mathrm{Ce})$ detector to $\gamma$-rays in the energy range 356-10190 keV. The data for low-energy photons were taken with the standard $\gamma$-ray sources, ${ }^{60} \mathrm{Co},{ }^{133} \mathrm{Ba},{ }^{137} \mathrm{Cs}$, and ${ }^{152} \mathrm{Eu}$, along with an Am-Be source. The data include the single escape peak of $4.4 \mathrm{MeV} \gamma$-rays (Am-Be). The data for 8-10 MeV $\gamma$-rays were taken with LCS $\gamma$-ray beams produced with a Nd: $\mathrm{YVO}_{4}$ laser. The solid line is the second-order polynomial fit to the data, while the dashed line is the best linear fit to the low-energy data excluding those for the LCS $\gamma$-rays.

$\gamma$-rays. These energy resolutions are less than those $(2.8 \%$ at $662 \mathrm{keV}, 2.1 \%$ at $1332 \mathrm{keV}$, and $1 \%$ at $10 \mathrm{MeV}$ ) reported in small (3 $\mathrm{mm}$ in diameter $\times 10 \mathrm{~mm}$ in length [16], $\left.2^{\prime \prime} \times 2^{\prime \prime}[17]\right)$ detectors.

Results of the Monte Carlo simulation are shown by the dotted lines in Fig. 7. The high-energy portion of the spectra including the FE peak and the bump of Compton scattering was well reproduced. It is noted that the low energy yield of the data includes contributions from backscattering by $\mathrm{Pb}$ blocks that were used as a part of the detector mount for the $\operatorname{LaBr}_{3}(\mathrm{Ce})$. The discrepancy between the experimental data and the simulation in the low-energy region is due to this backscattering. The FE peaks formed at $10.19,9.14$, and $8.19 \mathrm{MeV}$ corresponded to the channel numbers, 947.2, 857.8, and 775.8, respectively. The linearity in the response of the $\mathrm{LaBr}_{3}(\mathrm{Ce})$ detector to the 8 $-10 \mathrm{MeV}$ LCS $\gamma$-rays is shown in Fig. 8, where the low-energy data were taken with the standard $\gamma$-ray sources $\left({ }^{60} \mathrm{Co},{ }^{133} \mathrm{Ba}\right.$, ${ }^{137} \mathrm{Cs}$, and ${ }^{152} \mathrm{Eu}$ ) and an $\mathrm{Am}-\mathrm{Be}$ neutron source as a $4.4 \mathrm{MeV}$ $\gamma$-ray emitter, including the single escape peak.

The data show that the linearity is slightly deteriorated at high energies beyond the $\mathrm{Am}-\mathrm{Be}$ data at $4.4 \mathrm{MeV}$. A best fit with the second-order polynomial function is shown by the solid line in Fig. 8 together with the best-fit parameters. For comparison, a linear fit to the data taken with the standard $\gamma$-ray sources and the $\mathrm{Am}-\mathrm{Be}$ source is also shown by the dashed line in the figure. It is seen that the output of the $\mathrm{LaBr}_{3}(\mathrm{Ce})$ detector for $8-10 \mathrm{MeV}$ $\gamma$-rays is quenched to lower channel numbers from the linear response; the higher the energy is, the larger the quenching. A similar quenching effect was previously reported above $10 \mathrm{MeV}$ in a $2^{\prime \prime} \times 2^{\prime \prime} \mathrm{LaBr}_{3}(\mathrm{Ce})$ detector [17]. It is unknown, however, that the quenching is due to scintillation property intrinsic to the $\mathrm{LaBr}_{3}(\mathrm{Ce})$ crystal or saturation of the photomultiplier tube.

\section{B. Energy Profile of the LCS $\gamma$-ray Beams}

The present large volume $\mathrm{LaBr}_{3}(\mathrm{Ce})$ detector identified the FE peak in the response function as shown in Fig. 7. The Monte Carlo simulation, which best reproduced the response functions

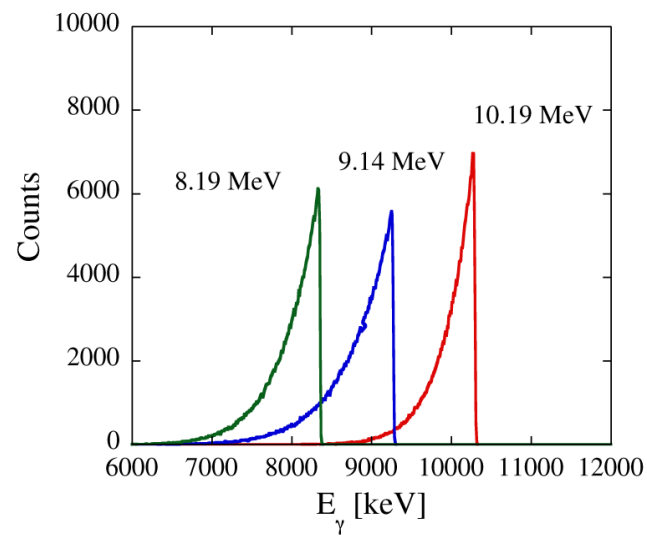

Fig. 9. Results of the Monte Carlo simulations for energy distributions of the LCS $\gamma$-ray beams produced at the $\gamma$-ray experimental hutch GACKO of the synchrotron radiation facility NewSUBARU.

of the $\mathrm{LaBr}_{3}(\mathrm{Ce})$ detector, also provides the energy distribution of the LCS $\gamma$-ray beam incident on the detector. The energy profile of the LCS $\gamma$-ray beams is shown in Fig. 9. The present study shows that using a 2-mm collimator, LCS -ray beams were produced at $10.19 \mathrm{MeV}$ in the FE peak with a $267 \mathrm{keV}$ spread $(\delta \mathrm{E})$ in FWHM, at $9.14 \mathrm{MeV}$ with $\delta \mathrm{E}=394 \mathrm{keV}$, and at $8.19 \mathrm{MeV}$ with $\delta \mathrm{E}=339 \mathrm{keV}$. The energy spread amounts to $2.6-4.3 \%$ in FWHM. The energy spread of the LCS $\gamma$-ray beam depends on tuning the electron and laser beam optics besides the collimator size.

\section{CONCLUSION}

We have systematically calibrated energies of the electron beams in the storage ring NewSUBARU in the nominal energy range of 550-974 MeV by using low-energy LCS $\gamma$-ray beams produced with a $\mathrm{CO}_{2}$ laser at the $\gamma$-ray experimental hutch GACKO. The absolute energy calibration of the electron beams with the high accuracy and excellent reproducibility provides a standard for the energy calibration of high-energy LCS $\gamma$-ray beams produced with a Nd: $\mathrm{YVO}_{4}$ laser. The energy-calibrated LCS $\gamma$-ray beams produced in the range of 5.6-17.3 MeV in the fundamental mode of the laser operation with $\lambda=1064 \mathrm{~nm}$ offer a variety of experimental opportunities in nuclear science. The energy range can be extended to 11.2-34.6 MeV and 16.8-51.9 MeV in the second and third harmonics of the laser with $\lambda=532 \mathrm{~nm}$ and $\lambda=355 \mathrm{~nm}$, respectively. As applications of the calibrated electron beams, we demonstrated a detector-calibration measurement with a $3.5^{\prime \prime} \times 4.0^{\prime \prime} \mathrm{LaBr}_{3}(\mathrm{Ce})$ detector in its response to $8-10 \mathrm{MeV}$ $\gamma$-rays and an energy-profile study of the high-energy LCS $\gamma$-ray beams. The large-volume $\operatorname{LaBr}_{3}(\mathrm{Ce})$ detector with the excellent response function and the good energy resolution can be a good instrument to determine the energy profile of the high-energy LCS $\gamma$-ray beams.

\section{ACKNOWLEDGMENT}

The authors would like to thank H. Ohgaki of the Institute of Advanced Energy, Kyoto University, for making a large volume $\mathrm{LaBr}_{3}(\mathrm{Ce})$ detector available to the present experiment. 


\section{REFERENCES}

[1] S. Amano, K. Horikawa, K. Ishihara, S. Miyamoto, T. Hayakawa, T. Shizuma, and T. Mochizuki, "Several-MeV $\gamma$-ray generation at NewSUBARU by laser Compton backscattering," Nucl. Instrum. Methods Phys. Res. A, vol. 602, pp. 337-341, 2009.

[2] K. Horikawa, S. Miyamoto, S. Amano, and T. Mochizuki, "Measurements for the energy and flux of laser Compton scattering -ray photons generated in an electron storage ring: NewSUBARU," Nucl. Instrum. Methods Phys. Res. A, vol. 618, pp. 209-215, 2010.

[3] A. Ando, S. Amano, S. Hashimoto, H. Kinosita, S. Miyamoto, T. Mochizuki, M. Niibe, Y. Shoji, M. Terasawa, T. Watanabe, and N. Kumagai, "Isochronous storage ring of the NewSUBARU project," $J$. Synchrotron Rad., vol. 5, pp. 342-344, 1998.

[4] G. Audi, A. H. Wapstra, and C. Thibault, "The AME2003 atomic mass evaluation (II). Tables, graphs and references," Nucl. Phys. A, vol. 729, pp. 337-676, 2003.

[5] L. C. Hsu, C.-C. Ching, and C.-I. Yu, "Energy measurement of relativistic electron beams by laser Compton scattering," Phys. Rev. E, vol. 54, pp. 5657-5663, 1996.

[6] R. Klein, T. Mayer, P. Kuske, R. Thornagel, and G. Ulm, "Beam diagnostics at the BESSY I electron storage ring with Compton backscattered laser photons: Measurement of the electron energy and related quantities," Nucl. Instrum. Methods in Phys. Res. A, vol. 384 , pp. 293-298, 1997.

[7] R. Klein, P. Kuske, R. Thornagel, G. Brandt, R. Görgen, and G. Ulm, "Measurement of the BESSY II electron beam energy by Comptonbackscattering of laser photons," Nucl. Instrum. Methods Phys. Res. A, vol. 486, pp. 545-551, 2002.
[8] H. Ohgaki, H. Toyokawa, K. Kudo, N. Takeda, and T. Yamazaki, "Generation and application of Laser-Compton gamma-ray at ETL," Nucl. Instrum. Methods Phys. Res. A, vol. 455, pp. 54-59, 2000.

[9] K. Chouffani, F. Harmon, D. Wells, J. Jones, and G. Lancaster, "Determination of electron beam parameters by means of laser-Compton scattering," Phys. Rev. ST Accel. Beams, vol. 9, pp. 050701-1-050701-11, 2006.

[10] C. Sun, J. Li, G. Rusev, A. P. Tonchev, and Y. K. Wu, "Energy and energy spread measurements of an electron beam by Compton scattering method," Phys. Rev. ST Accel. Beams, vol. 12, pp. 062801-1-062801-12, 2009.

[11] C. K. N. Patel, "Selective excitation through vibrational energy transfer and optical maser action in $\mathrm{N}_{2}-\mathrm{CO}_{2}$," Phys. Rev. Lett., vol. 13, p. 617,1964

[12] R. L. Abrams, "Broadening coefficients for the $\mathrm{P}(20) \mathrm{CO}_{2}$ laser transition," Applied Physics Letters, vol. 25, p. 609, 1974.

[13] Parameters of the NewSUBARU electron storage ring [Online]. Available: http://www.lasti.u-hyogo.ac.jp/NS-en/facility/ring/para.html

[14] W. R. Nelson, H. Hirayama, and W. O. Roger, "The EGS4 code systems," SLAC National Accelerator Lab., Menlo Park, CA, USA, SLAC-Report-265, 1985.

[15] H. Ohgaki, Feb. 27, 2014, private communications.

[16] E. V. D. van Loef, P. Dorenbos, C. W. E. van Eijk, K. Krämer, and H. U. Güdel, "High-energy-resolution scintillator: $\mathrm{Ce}^{3+}$ activated $\mathrm{LaBr}_{3}$," Appl. Phys. Lett., vol. 79, pp. 1573-1575, 2001.

[17] M. Ciemala, D. Balabanski, M. Csatlós, J. M. Daugas, G. Georgiev, J. Gulyás, M. Kmiecik, A. Krasznahorkay, S. Lalkovski, A. LefebreSchuhl, R. Lozeva, A. Maj, and A. Vitez, "Measurements of high-energy $\gamma$-rays with $\mathrm{LaBr}_{3}$ : Ce detectors," Nucl. Instrum. Methods Phys. Res. A, vol. 608, pp. 76-79, 2009. 